\title{
Determinação do Máximo Déficit Acumulado de Oxigênio: Efeito da Duração dos Testes Submáximos para Predição da Demanda de Oxigênio
}

\section{Determination of the Maximum Accumulated Oxygen Deficit: Effects of the Submaximal Tests Duration for Prediction of Oxygen Demand}

\author{
Henrique Bortolotti ${ }^{1}$ \\ Leandro R. Altimari ${ }^{1}$ \\ Fábio Y. Nakamura' \\ Eduardo B. Fontes ${ }^{2}$ \\ Alexandre H. Okano ${ }^{3}$ \\ Mara P. T. Chacon-Mikahil ${ }^{2}$ \\ Antonio C. Moraes² \\ Edilson S. Cyrino
}

1. Centro de Educação Física e Esporte. Universidade Estadual de Londrina, Londrina, PR, Brasil.

2. Faculdade de Educação Física. Universidade Estadual de Campinas, Campinas, SP, Brasil,

3. Departamento de Educação Física, Centro de Ciências da Saúde. Universidade Federal do Rio Grande do Norte,Natal, RN, Brasil.

\section{Endereço para correspondência:} Leandro Ricardo Altimari

Departamento de Educação Física Universidade Estadual de Londrina Rodovia Celso Garcia Cid, Pr 445 Km 380 - Campus Universitário, Cx. Postal 6001 - CEP 86051-990, Londrina, PR, Brasil.

E-mail: altimari@uel.br

\begin{abstract}
RESUMO
O objetivo do presente estudo foi verificar a influência de diferentes tempos de análise dos testes submáximos para determinação do máximo déficit acumulado de oxigênio (MAOD), adotando diferentes janelas de tempo 4-6min, 6-8min e 8-10min. Participaram do estudo 10 ciclistas com idade média de $27,5 \pm 4,1$ anos, massa corporal 74,4 $\pm 12,7 \mathrm{~kg}$ e tempo médio de prática de 9,8 $\pm 4,7$ anos. Os atletas realizaram um teste de esforço progressivo para determinação do consumo de oxigênio de pico $\left(\mathrm{VO}_{2}\right.$ pico) e quatro testes retangulares submáximos (60, 70, 80 e 90\% VO ${ }_{2}$ pico) com 10min de duração para estimar os valores da demanda de $\mathrm{O}_{2}\left(\mathrm{DEO}_{2}\right)$. Os valores médios de $\mathrm{VO}_{2}$ obtidos nas cargas para o tempo 4-6min, 6-8min e 8 a 10min foram aplicados em uma regressão linear entre a intensidade e o consumo de $\mathrm{O}_{2}$ para cada janela de tempo. Os sujeitos realizaram ainda um teste retangular supramáximo $\left(110 \% \mathrm{VO}_{2}\right.$ pico) para a quantificação do MAOD. Não foi constatada nenhuma diferença significativa no $\mathrm{VO}_{2}$ entre os diferentes períodos de tempo dos testes submáximos $(P>0,05)$. Da mesma forma, nenhuma diferença significativa foi constatada no $\mathrm{DEAO}_{2}$ e MAOD nos diferentes períodos de análise $(P>0,05)$. Verificou-se ainda que os valores de MAOD obtidos nos três intervalos de tempo apresentaram boa concordância e forte correlação. Dessa forma, os dados sugerem que os testes submáximos utilizados para gerar os valores do MAOD podem ser reduzidos ao menos nesse tipo de amostra e com a utilização de ciclossimulador.
\end{abstract}

Palavras-chave: déficit de oxigênio, capacidade anaeróbia, exercício supramáximo.

\begin{abstract}
The aim of this study was to investigate the influence of different assessment time periods of submaximal tests on the determination of the maximal accumulated oxygen deficit (MAOD), through the adoption of different time slots of 4 to 6,6 to 8 and 8 to $10 \mathrm{~min}$. Ten cyclists with mean age of $27.5 \pm 4.1$ years, body mass $74.4 \pm 12.7 \mathrm{~kg}$ and time experience of $9.8 \pm 4.7$ years participated in this study. The athletes underwent an incremental exercise test to determine the peak oxygen consumption $\left(\mathrm{VO}_{2}\right.$ peak), and four submaximal constant work-load test sessions $\left(60,70,80\right.$ and $90 \% \mathrm{VO}_{2}$ peak) of 10 min in order to estimate the $\mathrm{O}_{2}$ demand (DEO2). The mean $\mathrm{VO}_{2}$ values obtained on each constant work-load for the 4 to 6,6 to 8 and 8 to 10 min time-periods intervals were used to perform a linear regression between the intensity and $\mathrm{O}_{2}$ consumption for each time-period. In addition, the subjects performed one supramaximal rectangular test $\left(110 \% \mathrm{VO}_{2}\right.$ peak) for the quantification of MAOD. There was no significant difference in $\mathrm{VO}_{2}$ between the different time-periods for all submaximal tests $(P>0.05)$. Similarly, no significant difference was found in $D_{E A O_{2}}$ and $M A O D(P>$ 0.05). Furthermore, the values of MAOD for the three time-periods intervals showed good agreement and strong correlation. Thus, the data suggest that the submaximal tests used to estimate the values of MAOD can be reduced, at least in this type of sample, and with the use of a cycle simulator.
\end{abstract}

Keywords: oxygen deficit, anaerobic capacity, supramaximal exercise.

\section{INTRODUÇÃO}

$\mathrm{O}$ máximo déficit acumulado de $\mathrm{O}_{2}$ (MAOD) foi proposto originalmente por Medbo et al.(1) com o intuito de avaliar a capacidade anaeróbia. Desde então, diante da inexistência de um método padrão ouro para avaliação da contribuição energética do metabolismo anaeróbio no exercício, o MAOD tem sido considerado como a medida mais aceita da transferência de energia a partir das fontes aláticas e da glicólise anaeróbia ${ }^{(2,3)}$. 
Diante da necessidade de realização de grande quantidade de testes presente no método original e, consequentemente, de maior necessidade de disponibilidade de tempo por parte do avaliado, o método tem se tornado pouco aplicável(2), restringindo a medida apenas a laboratórios. Assim, alguns ajustes metodológicos têm sido recomendados na tentativa de melhorar a aplicabilidade da determinação do MAOD. Nesse sentido, diferentes protocolos ${ }^{(1)}$ têm proposto alterações tanto na quantidade de testes submáximos ${ }^{(1,4-6)}$ quanto na duração desses testes ${ }^{(6-13)}$.

Nesse sentido, Gardner et al. ${ }^{(14)}$ não constataram quaisquer diferenças significativas entre os valores do MAOD de ciclistas treinados, os quais foram gerados por testes submáximos que tiveram o tempo de análise do $\mathrm{VO}_{2}$ entre 1 e $3 \mathrm{~min}$, 4 e 6 min ou 8 e 10min, obtidos por cinco testes de cargas constantes. Dessa forma, esses ajustes metodológicos podem prover maior aplicabilidade ao método. Resultados diferentes foram apresentados por Buck e McNaughton ${ }^{(15)}$, que mostraram haver diferenças significativas entres os intervalos 2-4min e 8-10min, utilizados para a determinação do MAOD. Assim, parece não estar claro qual o intervalo de tempo mínimo adequado para a determinação do MAOD. Cabe ressaltar que, em ambos os estudos, não foi assumido o procedimento 3 de Medbo et al. ${ }^{(1)}$, que consiste na adoção de um intercepto y comum a todos os indivíduos de $5 \mathrm{ml}^{.} \mathrm{kg}^{-1} \cdot \mathrm{min}^{-1}$. Diante disso, seria interessante investigar a utilização de diferentes intervalos de tempo assumindo esse procedimento, que permite uma redução no número de testes submáximos.

Nesse sentido, o presente estudo teve como objetivo verificar a influência de diferentes tempos de análise dos testes submáximos para determinação do MAOD, adotando diferentes janelas de tempo: 4-6min, 6-8min e 8-10min.

\section{MATERIAL E MÉTODOS}

\section{Seleção da amostra}

Inicialmente, 14 ciclistas amadores na faixa etária de 20 a 32 anos, do sexo masculino, consentiram em participar deste estudo. Como critério inicial de inclusão, os indivíduos deveriam ter experiência mínima de dois anos em competição. Quatro desses sujeitos acabaram não concluindo o experimento por diversos motivos, incluindo abandono voluntário (1), não-cumprimento de procedimentos estabelecidos previamente ao início do estudo (1), problemas de saúde não relacionados ao protocolo experimental, ou problemas particulares (2).

Desse modo, a amostra investigada foi composta por 10 ciclistas (velocistas $=3$ e endurance $=7$ ) com idade média de 27,5 \pm 4,1 anos e tempo médio de prática na modalidade de 9,8 $\pm 4,7$ anos. Todos relataram participar de treinamento em média 4,6 \pm 1,3 vezes por semana, mantendo volume médio semanal de $364 \pm 76 \mathrm{~km}$. Os indivíduos foram convenientemente informados sobre a proposta do estudo e sobre os procedimentos aos quais seriam submetidos e, posteriormente, assinaram o Termo de Consentimento Livre e Esclarecido. Este estudo foi aprovado pelo Comitê de Ética em Pesquisa da Faculdade de Ciências Médicas da Universidade Estadual de Campinas (Parecer 228/2005), de acordo com as normas da Resolução 196/96 do Conselho Nacional de Saúde sobre pesquisa envolvendo seres humanos.

\section{Delineamento experimental}

O delineamento experimental adotado teve como intuito controlar variáveis que pudessem exercer influência na investigação, possibilitando análise mais criteriosa dos achados. Portanto, as variáveis temperatura ambiente e umidade relativa do ar foram mantidas entre 21 e $24^{\circ} \mathrm{C}$ e 40 e $60 \%$, respectivamente.

O presente estudo foi dividido em três etapas, realizadas no período de três a quatro semanas. Na primeira etapa do experimento, os atletas compareceram ao laboratório para tomarem conhecimento da proposta do estudo e procedimentos aos quais seriam submetidos. Nesse encontro, todos os esclarecimentos foram feitos. Em seguida, foram realizadas medidas antropométricas para caracterização da amostra e teste pré-experimental, no intuito de familiarizar os sujeitos aos equipamentos e aos protocolos. Vale ressaltar que todos os testes foram realizados com intervalo mínimo de 72 horas entre eles.

Na segunda etapa do experimento, os voluntários compareceram ao laboratório nos horários pre-definidos, onde foram submetidos ao teste de esforço progressivo para determinação do consumo de oxigênio de pico ( $\mathrm{VO}_{2}$ pico). Na etapa seguinte, os sujeitos compareceram ao laboratório em duas sessões distintas, onde foram submetidos aos testes retangulares submáximos (60, 70, 80 e 90\% da carga referente ao $\mathrm{VO}_{2}$ pico) utilizados para determinar o consumo de oxigênio durante esforços de intensidades submáximas.

Na terceira etapa, os sujeitos compareceram novamente ao laboratório, onde foram submetidos ao teste retangular supramáximo (110\% do $\mathrm{VO}_{2}$ pico) para a quantificação do déficit máximo acumulado de oxigênio (MAOD).

Os horários de avaliação de cada voluntário foram padronizados entre os dias de testes, fazendo com que estes realizassem todos os testes em horário aproximado, evitando assim possíveis interferências circadianas. Vale ressaltar que os sujeitos foram orientados para que não realizassem atividades físicas vigorosas nas 24h, e não ingerissem substâncias cafeinadas (café, chocolate, mate, pó-de-guaraná, coca-cola e guaraná) ou alcoólicas nas 48h precedentes aos testes, para evitar possíveis interferências.

\section{Antropometria}

A massa corporal (MC) foi medida em balança da marca Filizola, modelo ID-1500, com precisão de 0,1 kg, ao passo que a estatura (E) foi obtida em antropômetro, com precisão de 0,1cm, de acordo com os procedimentos descritos por Gordon et al. ${ }^{(16)}$. Todos os indivíduos foram medidos descalços, vestindo apenas uma sunga.

\section{Teste de esforço progressivo}

Os indivíduos realizaram teste de esforço progressivo (rampa) de 20W. min $^{-1}$ em ciclossimulador modelo CompuTrainer DYNAFIT ${ }^{\mathrm{M}}$ (RacerMate ${ }^{\circledR}$, Seattle, WA, EUA), mantendo cadência de 90 rotações por minuto (rpm) até a exaustão voluntária; o mesmo teve início com aquecimento de três minutos em carga de 50W. Como critério de interrupção do teste, adotou-se a queda na cadência de rotações (90rpm) sem recuperação, por mais de cinco segundos, a despeito do incentivo verbal. O consumo de oxigênio de pico $\left(\mathrm{VO}_{2}\right.$ pico) foi determinado considerando a média dos dois maiores valores consecutivos obtidos em intervalo de 15s. As medidas da bicicleta correspondente a cada avaliado como: altura e distância do banco; altura e distância da mesa e a posição das mãos, foram padronizadas durante todos os testes.

\section{Determinação do déficit máximo acumulado de oxigênio (MAOD)}

Para determinação do MAOD, cada participante foi submetido a quatro sessões de testes retangulares submáximos (60, 70, 80 e 90\% da carga referente ao $\mathrm{VO}_{2}$ pico) com 10 minutos de duração, mantendo uma cadência de 90rpm, com o objetivo de estimar os valores da demanda de $\mathrm{O}_{2}\left(\mathrm{DEO}_{2}\right)$. Os valores médios de $\mathrm{VO}_{2}$ obtidos nas cargas acima foram registrados para os momentos: 4-6min, 6-8min e 8-10 min, para a construção de uma regressão linear entre a intensidade e o consumo de $\mathrm{O}_{2}$ para cada janela de tempo (figura 1).

Os testes retangulares submáximos foram realizados em duas sessões distintas, com intervalo de 48 horas entre as mesmas, sendo 


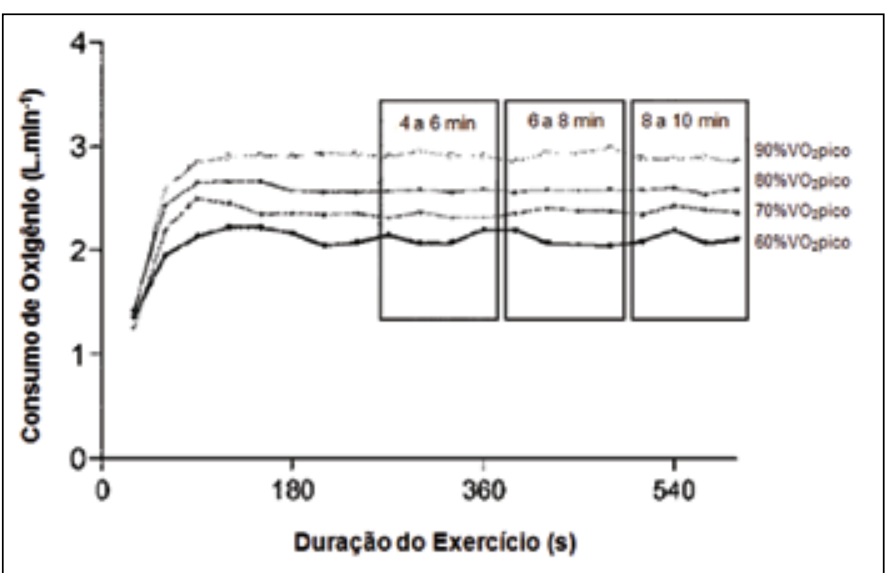

Figura 1. Representação esquemática dos diferentes intervalos utilizados para determinação do MAOD.

executados dois testes $\left(70,80 \%\right.$ do $\mathrm{VO}_{2}$ pico) na primeira sessão e outros dois (60 e $90 \%$ do $\mathrm{VO}_{2}$ pico) na segunda sessão. A ordem da execução das sessões de testes foi aleatória dentro da sessão e o intervalo entre os testes foi de pelo menos 30 minutos.

Com exceção da imposição da resistência, todos os procedimentos adotados nos testes retangulares submáximos foram idênticos ao teste de esforço progressivo. As cargas empregadas tiveram potência média compreendida entre 176 e $284 \mathrm{~W}$.

Posteriormente, utilizando-se os valores de $\mathrm{DEO}_{2}$ respectivos a cada intensidade de esforço, foi estabelecida uma relação linear entre $\mathrm{DEO}_{2}$ e intensidade e, em seguida, foi estimada a demanda acumulada de $\mathrm{O}_{2}$ $\left(\mathrm{DEAO}_{2}\right)$ em esforços supramáximos ${ }^{(14)}$. Para tanto, foi utilizado o procedimento 3 de Medbo et al. ${ }^{(1)}$, que consiste na adoção de um intercepto y comum a todos os indivíduos, de $5 \mathrm{ml} . \mathrm{kg}^{1} \cdot \mathrm{min}^{-1}$, para melhorar a aplicabilidade do MAOD (diminuição do número de testes submáximos). A extrapolação desse segmento de reta para intensidades supra- $\mathrm{VO}_{2}$ pico fornece o valor da $\mathrm{DEAO}_{2}$ para o teste retangular de MAOD.

Por fim, para estimativa do MAOD, foi realizado teste retangular supramáximo (intervalo mínimo de 72 horas dos testes submáximos) com carga correspondente a $110 \%$ do $\mathrm{VO}_{2}$ pico até a exaustão voluntária, mantendo sempre uma cadência de 90rpm.

Como critério de interrupção do teste, adotou-se o valor limite 85rpm, sem recuperação por mais de cinco segundos. 0 consumo de oxigênio acumulado $\left(\mathrm{AVO}_{2}\right)$ no teste supramáximo foi determinado a partir da integral da curva de $\mathrm{VO}_{2}$ ao longo do teste, conforme procedimento utilizado por Hirai et al. ${ }^{(17)}$. O MAOD foi quantificado pela diferença entre a $\mathrm{DEAO}_{2}$ estimada para o teste supramáximo e o $\mathrm{AVO}_{2}$ no teste supramáximo.

Durante os testes supramáximos, os voluntários receberam forte encorajamento verbal para garantir que alcançassem seu desempenho máximo. Além disso, não foi dada informação aos participantes quanto à intensidade das cargas e duração esperada dos testes.

\section{Ergoespirometria}

Para todos os testes, os parâmetros respiratórios foram medidos continuamente em sistema de ergoespirometria (CPX/D MedGraphics ${ }^{\mathrm{TM}}$, Breeze ${ }^{\circledR}$, St. Paul, MN, EUA), utilizando-se da técnica breath-by-breath. 0 equipamento foi calibrado antes de cada teste de acordo com a padronização do fabricante, utilizando-se seringa de calibração de três litros Hans Rudolf 5530 (figura 6B) e mistura de gás de $16 \% \mathrm{O}_{2}$ com balanço de $5 \% \mathrm{CO}_{2}$ e $95 \% \mathrm{~N}_{2}$ (White Martins ${ }^{\circledR}$, Campinas, SP, Brasil).

\section{Análise estatística}

Para análise dos resultados, foi empregado o pacote estatístico Statistica $^{\mathrm{TM}} 6.0^{\circledR}$ (Statsoft Inc., Tulsa, OK, EUA). Após constatação da normalidade (teste de Shapiro-Wilk) e homogeneidade (teste de Levene), os resultados obtidos no estudo foram agrupados em valores de média e desvio padrão. As comparações dos parâmetros medidos na determinação do déficit máximo acumulado de oxigênio $\left(\mathrm{DEAO}_{2}, \mathrm{e}\right.$ MAOD) foram realizadas utilizando-se do teste ANOVA one-way. Além disso, o coeficiente de correlação linear de Pearson e análise de concordância, proposta por Bland e Altman (1986), foram utilizados. O nível de significância adotado para todas as análises foi de 5\%.

\section{RESULTADOS}

Na tabela1 estão representadas as características antropométricas e os parâmetros fisiológicos dos sujeitos da amostra.

Tabela 1. Média \pm desvio padrão das características antropométricas e parâmetros fisiológicos dos sujeitos.

\begin{tabular}{c|c}
\hline Massa corporal (kg) & $74,4 \pm 12,7$ \\
\hline FC $_{\text {max }}(\mathrm{bpm})$ & $197,3 \pm 6,9$ \\
\hline VO2pico (ml.kg-1.min-1) & $56,9 \pm 5,8$ \\
\hline VO2pico (ml.min-1) & $4.132,0 \pm 479,1$ \\
\hline Potência pico (W) & $355,8 \pm 43,8$ \\
\hline
\end{tabular}

Os valores médios de $\mathrm{VO}_{2}$ referentes às quatro intensidades submáximas, $\mathrm{DEAO}_{2}$ e MAOD, assim como os valores de $\mathrm{F}$ e $P$ estão apresentados na tabela 2. Não foi constatada nenhuma diferença significativa no $\mathrm{VO}_{2}$ entre os diferentes períodos de tempo para todos os testes submáximos $(P>0,05)$. Da mesma forma, nenhuma diferença significativa foi constatada no $\mathrm{DEAO}_{2}$ e MAOD nos diferentes períodos de tempo $(P>0,05)$.

Tabela 2. Média \pm desvio padrão, valores de consumo de oxigênio nos quatro testes de intensidades submáximas, DEAO2 e MAOD, utilizando os intervalos de tempo 4-6min, 6-8min e 8-10min.

\begin{tabular}{c|c|c|c|c|c}
\hline & $\mathbf{4 - 6 m i n}$ & $\mathbf{6 - 8 m i n}$ & $\mathbf{8 - 1 0 m i n}$ & $\mathbf{F}$ & $\mathbf{P}$ \\
\hline VO2 (ml.min-1) & & & & & \\
\hline $60 \%$ VO2pico & $2.609,9 \pm 146,3$ & $2.577,4 \pm 118,6$ & $2.560,0 \pm 163,8$ & 0,39 & 0,67 \\
\hline $70 \%$ VO2pico & $2.932,3 \pm 300,8$ & $2.908,3 \pm 292,7$ & $2.883,2 \pm 277,4$ & 0,12 & 0,88 \\
\hline $80 \%$ VO2pico & $3.276,0 \pm 402,4$ & $3.279,5 \pm 381,0$ & $3.273,0 \pm 372,8$ & 0 & 0,99 \\
\hline $90 \%$ VO2pico & $3.730,0 \pm 517,8$ & $3.783,6 \pm 530,9$ & $3.817,5 \pm 535,5$ & 0,09 & 0,91 \\
\hline DEAO2 (L) & $9,74 \pm 2,21$ & $9,93 \pm 2,21$ & $9,78 \pm 2,28$ & 0,02 & 0,97 \\
\hline MAOD $(\mathrm{L})$ & $5,91 \pm 2,09$ & $6,09 \pm 2,12$ & $5,98 \pm 2,16$ & 0,02 & 0,98 \\
\hline
\end{tabular}

$\mathrm{DEAO}_{2}=$ demanda acumulada de $\mathrm{O}_{2}, \mathrm{MAOD}=$ máximo déficit acumulado de $\mathrm{O}_{2}$

Através da análise de Bland-Altman (figura 2), verificou-se que os valores de MAOD obtidos nos três intervalos de tempo (4-6, 6-8 e 8-10min) apresentaram boa concordância. Ressalta-se ainda que foi observada forte correlação entre os valores de MAOD obtidos nos três intervalos de tempo (tabela 3).

Tabela 3. Valores de BIAS e limites (derivados da análise de Bland-Altman), coeficiente de correlação $(r)$ e coeficiente de determinação $\left(R^{2}\right)$.

\begin{tabular}{c|c|c|c}
\hline & BIAS & $\mathbf{r}$ & $\mathbf{R}^{\mathbf{2}}$ \\
\hline MAOD 4-6 vs. 6-8 & $-0,18(-1,28-0,91)$ & $0,96^{*}$ & 0,93 \\
\hline MAOD 4-6 vs. 8-10 & $-0,07(-0,49-0,35)$ & $0,99^{*}$ & 0,99 \\
\hline MAOD 6-8 vs. 8-10 & $0,11(-0,90-1,12)$ & $0,97^{*}$ & 0,94 \\
\hline
\end{tabular}


A

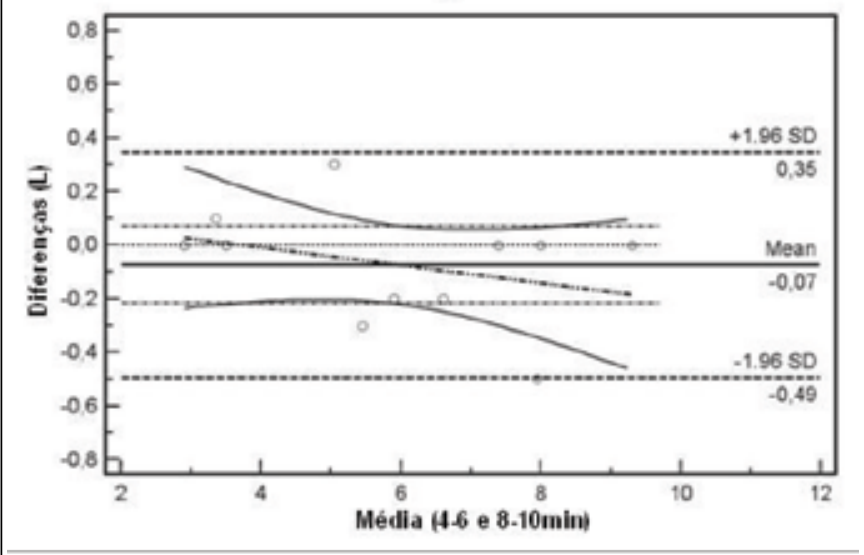

B
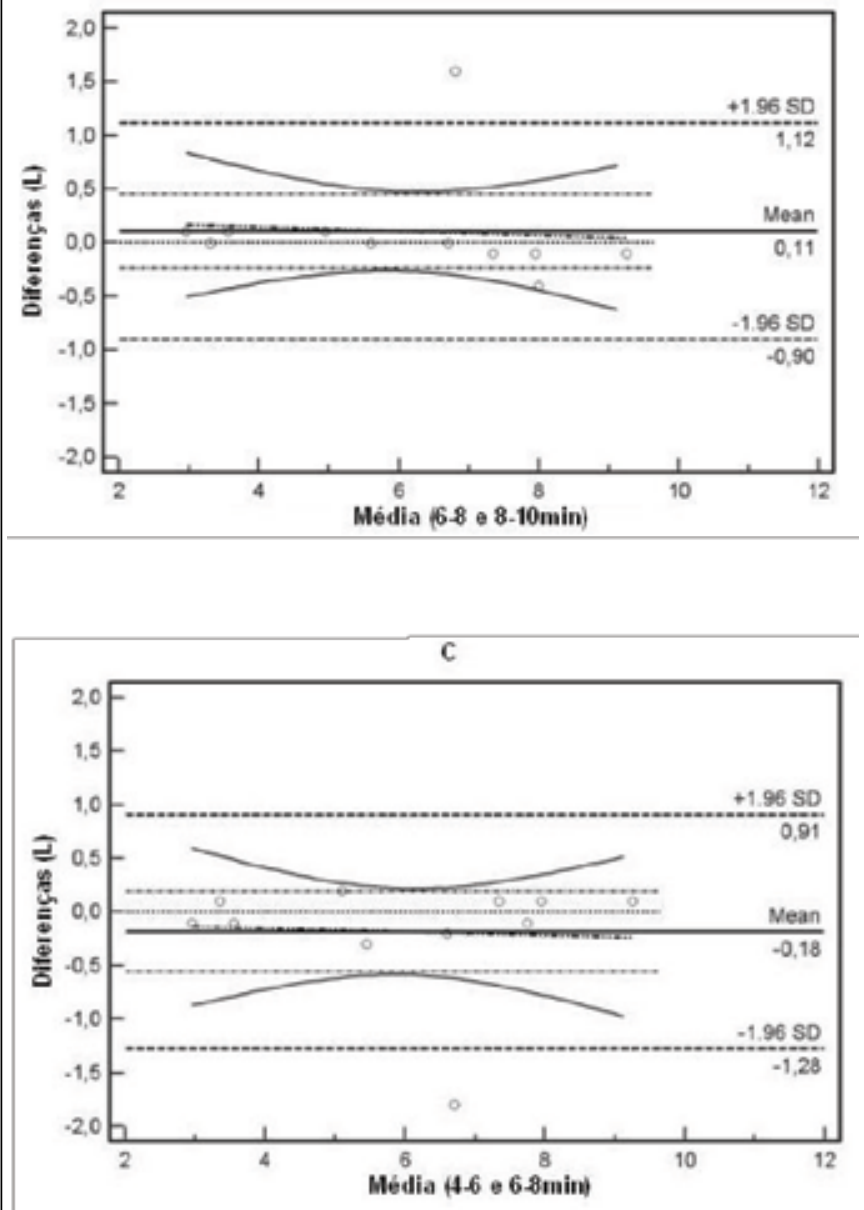

Figura 2. Representações da plotagem de Bland-Altman dos valores de MAOD obtidos nas condições 4-6 vs. 8-10 min (A), 6-8 vs. 8-10min (B) e 4-6 vs. 6-8min (C).

\section{DISCUSSÃO}

Na tentativa de aumentar a aplicabilidade da determinação do MAOD, algumas modificações têm sido propostas ao método original, diminuindo o número de testes submáximos ${ }^{(1,4-6)}$ e a duração dos testes $^{(6-13)}$. Desse modo, o propósito do presente estudo foi verificar a utilização de diferentes janelas de tempos (4-6, 6-8 e 8-10min) na determinação do MAOD.
A adoção do procedimento 3 de Medbo et al.(1), que consiste na adoção de um intercepto y comum a todos os indivíduos, de $5 \mathrm{ml}$. $\mathrm{kg}^{-1} \cdot \mathrm{min}^{-1}$, permite que menos testes submáximos sejam realizados, desde que os outros pontos disponíveis, de demanda de $\mathrm{O}_{2}$, incluam intensidades muito próximas ao $\mathrm{VO}_{2}$ pico.

Buck e McNaughton ${ }^{(15)}$ verificaram a influência da redução do tempo dos exercícios submáximos no cálculo do MAOD. Oito ciclistas foram submetidos a 10 testes com cargas que variaram entre 30 e $90 \%$ do $\mathrm{VO}_{2}$ pico, tendo duração de 10 minutos. Os valores de $\mathrm{VO}_{2}$ empregados na equação linear foram gerados com base na análise de diferentes intervalos de tempo (2-4, 6-8 e 8-10min). Quando comparados os valores do MAOD produzidos por meio da janela de tempo 8-10min, a janela 2-4min teve menor estimativa. Dessa forma, além de recomendarem pelo menos oito minutos de exercício submáximo, os autores concluíram que a utilização de períodos muito curtos de tempo pode ser inadequada para a determinação do MAOD. Possivelmente, esse achado pode ser creditado ao fato de o $\mathrm{VO}_{2}$ não depender apenas da intensidade empregada, mas também da duração das sessões submáximas, devido ao componente lento da cinética de $\mathrm{VO}_{2}$. Esta parece estar relacionada ao recrutamento de fibras musculares do tipo $\|^{(18)}$. Dessa forma, sua manifestação nas intensidades mais altas pode levar a um aumento da estimativa da $\mathrm{DEAO}_{2}$ e, consequentemente, aumentar a estimativa de MAOD com sessões submáximas mais longas. Entretanto, Gardner et al. ${ }^{(14)}$ não verificaram qualquer diferença significativa entre os valores do MAOD em outra amostra, também de ciclistas, gerados por testes submáximos que tiveram tempo de análise do $\mathrm{VO}_{2}$ entre 1-3, 4-6 e 8-10min. Demonstraram dessa forma que, possivelmente, o intervalo de tempo mais curto tenha sido suficiente para a estabilização do $\mathrm{VO}_{2}$ nas intensidades escolhidas para os testes submáximos (100-200W).

Esses achados parecerem contradizer os fenômenos relacionados à cinética de $\mathrm{VO}_{2}$ já mencionados. No entanto, sabe-se que o treinamento de endurance pode reduzir significativamente o componente lento do aumento de $\mathrm{VO}_{2}{ }^{(19)}$ mesmo em intensidades relativas altas em relação ao $\mathrm{VO}_{2} \mathrm{pico}^{(20)}$. Dessa forma, o estado de treinamento dos atletas parece ser fator determinante dos possíveis vieses causados pela manipulação da duração das cargas submáximas destinadas a estimar a $\mathrm{DAO}_{2}$.

A diminuição na duração dos testes submáximos pode viabilizar ainda mais a utilização do MAOD que, originalmente, utiliza testes submáximos de 10min de duração. Os resultados apresentados nos presente estudo corroboram os achados de Gardner et al. ${ }^{(14)}$, não havendo diferença no valor gerado de MAOD. Isso pode ser creditado ao estado de treinamento dos sujeitos deste estudo, ou até ao fato de nossos testes terem sido realizados em ciclossimulador. Esse fator poderia limitar a comparação com estudos prévios. Dessa forma, em estudos futuros, a cinética de $\mathrm{VO}_{2}$ poderia ser determinada em ambos ergômetros (cicloergômetro e ciclossimulador). Independente disso, demonstrou-se uma boa concordância entre as janelas de tempo escolhidas para análise (4-6, 6-8 e 8-10min).

\section{CONCLUSÃO}

Os intervalos de tempo utilizados na determinação do MAOD (4-6, 6-8 e 8-10min) não apresentaram diferenças entre si. Dessa forma, pode-se sugerir que os testes submáximos utilizados para gerar os valores do MAOD podem ser reduzidos para a presente amostra ao menos nesse tipo de amostra e com a utilização de ciclossimulador.

Todos os autores declararam não haver qualquer potencial conflito de interesses referente a este artigo. 


\section{REFERÊNCIAS}

1. Medbo Jl, Mohn AC, Tabata I, Bahr R, Vaage O, Sejersted OM. Anaerobic capacity determined by maximal accumulated O2 deficit. J Appl Physiol. 1988;64:50-60.

2. Bertuzzi RCM, Silva AEL, Pires FO, Kiss MAPDM. Déficit acumulado de oxigênio: uma breve revisão histórica e metodológica. Revista de Educação Física/UEM. 2008;19:131-44.

3. Nakamura FY, Franchini E. Máximo déficit acumulado de oxigênio como preditor de capacidade anaeróbia. Revista Brasileira de Cineantropometria \& Desempenho Humano. 2006;8:88-95.

4. Calbet JA, Chavarren J, Dorado C. Fractional use of anaerobic capacity during a 30- and a 45-s Wingate test. Eur J Appl Physiol Occup Physiol. 1997;76:308-13.

5. Levine BD, Stray-Gundersen J. "Living high-training low": effect of moderate-altitude acclimatization with low-altitude training on performance. J Appl Physiol. 1997;83:102-12.

6. Weber CL, Schneider DA. Increases in maximal accumulated oxygen deficit after high-intensity interval training are not gender dependent. J Appl Physiol. 2002;92:1795-801.

7. Bell DG, Jacobs I, Ellerington K. Effect of caffeine and ephedrine ingestion on anaerobic exercise performance. Med Sci Sports Exerc. 2001;33:1399-403.

8. Doherty M. The effects of caffeine on the maximal accumulated oxygen deficit and short-term running performance. Int J Sport Nutr. 1998;8:95-104.

9. Doherty M, Smith PM, Schroder K. Reproducibility of the maximum accumulated oxygen deficit and run time to exhaustion during short-distance running. J Sports Sci. 2000;18:331-8.

10. Hargreaves M, Finn JP, Withers RT, Halbert JA, Scroop GC, Mackay M, et al. Effect of muscle glycogen availability on maximal exercise performance. Eur J Appl Physiol Occup Physiol. 1997;75:188-92

11. Sloniger MA, Cureton KJ, O'Bannon PJ. One-mile run-walk performance in young men and women: role of anaerobic metabolism. Can J Appl Physiol. 1997;22:337-50.

12. Wadley $G$, Le Rossignol P. The relationship between repeated sprint ability and the aerobic and anaerobic energy systems. J Sci Med Sport. 1998;1:100-10.

13. Woolford SM, Withers RT, Craig NP, Bourdon PC, Stanef T, McKenzie I. Effect of pedal cadence on the accumulated oxygen deficit, maximal aerobic power and blood lactate transition thresholds of highperformance junior endurance cyclists. Eur J Appl Physiol Occup Physiol. 1999;80:285-91.

14. Gardner A, Osborne M, D'Auria S, Jenkins D. A comparison of two methods for the calculation of accumulated oxygen deficit. J Sports Sci. 2003;21:155-62.

15. Buck D, McNaughton L. Maximal accumulated oxygen deficit must be calculated using 10-min time periods. Med Sci Sports Exerc. 1999;31:1346-9.

16. Gordon CC, Chumlea WC, Roche AF. Stature, recumbent length, and weight. In: Lohman TG, Roche AF, Martorell $R$, editors. Anthropometric standardization reference manual. Champaign: Human Kinetic Books 1988:3-8.

17. Hirai DM, Okuno NM, Perandini LAB, Puga, GM, Simões, HG, Nakamura, FY. Cinética do consumo de oxigênio durante exercícios supramáximos: aplicação de modelos matemáticos. Revista Brasileira de Cineantropometria \& Desempenho Humano. 2008;10:43-9.

18. Barstow TJ, Jones AM, Nguyen PH, Casaburi R. Influence of muscle fiber type and pedal frequency on oxygen uptake kinetics of heavy exercise. J Appl Physiol. 1996;81:1642-50.

19. Casaburi R, Storer TW, Ben-Dov I, Wasserman K. Effect of endurance training on possible determinants of VO2 during heavy exercise. J Appl Physiol. 1987;62:199-207.

20. Billat V, Binsse V, Petit B, Koralsztein JP. High level runners are able to maintain a VO2 steady-state below $\mathrm{VO}_{\text {max }}$ in an all-out run over their critical velocity. Arch Physiol Biochem. 1998;106:38-45. 